\title{
eJRIEPS
}

Ejournal de la recherche sur l'intervention en éducation physique et sport

$35 \mid 2015$

Varia

\section{Approche technologique et forme de pratique scolaire du football en milieu difficile : le modèle $\mathrm{du}$ « futsal »}

\section{Guillaume Dietsch}

\section{(2) OpenEdition}

Édition électronique

URL : http://journals.openedition.org/ejrieps/1606

DOI : 10.4000/ejrieps.1606

ISSN : 2105-0821

Éditeur

ELLIADD

Référence électronique

Guillaume Dietsch, «Approche technologique et forme de pratique scolaire du football en milieu difficile : le modèle du « futsal » », eJRIEPS [En ligne], 35 | 2015, mis en ligne le 01 avril 2015, consulté le 04 octobre 2019. URL : http://journals.openedition.org/ejrieps/1606; DOI : 10.4000/ejrieps.1606

Ce document a été généré automatiquement le 4 octobre 2019.

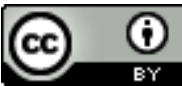

La revue eJRIEPS est mise à disposition selon les termes de la Creative Commons Attribution 4.0 International License. 


\title{
Approche technologique et forme de pratique scolaire du football en milieu difficile : le modèle du «futsal »
}

\author{
Guillaume Dietsch
}

Le football répond, comme tous les autres sports collectifs enseignés, aux objectifs de l'Education Physique et Sportive (EPS). Pourtant, les vertus éducatives de cette Activité Physique, Sportive et Artistique (APSA) semblent parfois décriées au regard des comportements déviants et violents présents dans le football et médiatisés dans la société. Cary et Bergez (2010), dans une étude ethnographique de deux clubs de football populaires amateurs en France, relèvent notamment de la part des joueurs un rapport problématique à la règle, voire un rejet de la norme provenant d'une "défiance généralisée envers l'ensemble des institutions supposées produire du lien social (école, association) ».

Les représentations négatives du football dans la société rejaillissent au niveau scolaire, où le « foot » subit le refus voire l'opprobre des enseignants d'EPS à le programmer et à l'enseigner. Comme en témoigne le Numéro Spécial sur le «Football» de la revue Contre-Pied, le football semble indésirable à l'école, "ce qui entraîne une "quasi disparition $d u$ "foot" des programmations d'établissement, des évaluations et examens nationaux» (Becker, 2014, p.4). Paradoxalement, l'activité football représente la première pratique institutionnelle sportive en France.

Les réticences des enseignants d'EPS à proposer l'activité football semblent renforcées en milieu difficile « citadin » au regard du rapport à l'activité des élèves. En effet, les élèves de «quartier" vouent un "culte de la performance individuelle " (Lepoutre, 1997). L'auteur relate ainsi le fait que «Dans les sports de rue pratiqués par les adolescents, que ce soit le football, le basket (...), la réussite se mesure tout autant à la démonstration des capacités physiques, aux prouesses, aux exploits et aux records de chacun qu'aux points accumulés ou à la partie gagnée » (Lepoutre, 1997, p. 398). Ainsi, la pratique du football s'inscrit dans une réalité sociale plus vaste puisqu'elle dépend dans une certaine 
mesure de la manière dont les acteurs perçoivent cette APSA. Le «football de pied d'immeuble » décrit par Travert (1997) montre la prééminence de l'individu sur le collectif. Ce type de joueur " perso » (Caillé, 2008) se singularise en réalisant l'exploit et en effectuant de beaux gestes.

L'impact médiatique du football est fort chez ces jeunes : «le sportif, dont le footballeur est le représentant le plus médiatisé en Occident, constitue l'un des principaux modèles de réussite sociale et économique» (Zotian, 2010, p. 4). Ces projections de soi en tant que footballeur professionnel sont liées au sentiment que l'école n'est pas (ou plus) la voie de l'ascension sociale (Zotian, 2010). Cette représentation est notamment significative chez certains garçons qui peuvent rencontrer d'importantes difficultés scolaires. L'orientation en lycée professionnel, qui est le plus souvent subie et pas réellement choisie pour nombre d'élèves issus le plus souvent de milieux défavorisés, est perçue comme une condamnation sociale par anticipation « qui voue au mieux au destin d'ouvrier et au pire à celui de chômeur » (Beaud, 2003).

Prenant appui sur des "pratiques sociales de référence» (Martinand, 1989), l'enseignant d'EPS se trouve confronté d'une part, à la question de la référence culturelle par rapport à la culture sportive et à la diversité des formes de pratique du football prises dans la société et dans les cités (Mauny \& Gibout, 2008). Or, nous observons actuellement une «transformation de la pratique $d u$ football dans les quartiers, notamment avec l'émergence du futsal » (Beaud \& Guimard, 2014, p. 222). Le futsal est né de la volonté de créer un nouveau sport collectif avec des règles du jeu spécifiques et adaptées aux missions d'une association de jeunes chrétiens en Uruguay. Il emprunta pour cela aux règles d'autres sports collectifs déjà codifiés (handball, basket-ball, water-polo) tout en conservant le but principal du football, de marquer des buts avec une balle au pied. L'idée originelle n'était pas simplement de jouer au football sur un plus petit terrain (intérieur ou extérieur), mais bel et bien de créer un nouveau sport avec ses propres règles beaucoup plus strictes, dans l' « esprit d'éduquer les jeunes adultes, de les responsabiliser et de les rendre solidaires" (Kievits-Boucher, 2006). Au même moment, l'utilisation des jeux réduits en football se développe en Europe (Frantz, 1975). D'autre part, la question de la référence renvoie à une diversité des modalités de pratique. Nous pouvons ainsi distinguer le «football de rue » et le "football en club " (Zotian, 2010). La pratique sociale de référence n'est donc plus la même.

Quelle pratique du football l'enseignant d'EPS peut-il alors transposer dans son contexte d'enseignement, afin de préserver ce qui semble légitime culturellement? L'enseignant est amené à faire des choix d'objets d'enseignement culturellement significatifs de l'APSA pour répondre aux objectifs scolaires. Pour sélectionner ces objets d'enseignement, il convient de ne pas se focaliser uniquement sur l'APSA en tant que pratique sociale, mais plutôt de se centrer sur l'activité de l'élève en train de vivre une expérience sportive originale. Dans le cadre de notre recherche, l'expérience aura du sens pour l'élève s'il s'intéresse à l'action collective, s'il y voit un intérêt personnel et enfin si les règles mises en place amènent un aspect positif dans leur pratique. Cette réflexion doit "aboutir à l'élaboration d'une forme de pratique scolaire permettant de faire vivre aux élèves une expérience sportive culturellement signifiante " (Mascret \& Dhellemmes, 2011, p. 99). 


\section{Cadre théorique}

2 Dans cette première partie, nous explicitons notre cadre théorique ainsi que nos questions de recherche. Nous nous inscrivons dans le cadre de l'approche technologique à visée didactique (Bouthier \& Durey, 1994).

\subsection{Rapport au savoir et formes de pratique scolaire en football}

3 Pour des jeunes issus de milieu difficile où l'école ne joue plus le rôle d'ascenseur social, la question du sens devient centrale (Dubet \& Duru-Bellat, 2000). Pour l'équipe ESCOL (Education, Scolarisation) et le réseau RESEIDA (Recherches sur la Socialisation, l'Enseignement, les Inégalités et les Différenciations dans les Apprentissages), elle permet de mieux comprendre le lien entre le sujet et le savoir. Les auteurs définissent la notion de rapport au savoir comme "une relation de sens, et donc de valeur, entre un individu (ou un groupe) et les processus ou produits du savoir " (Charlot, Bautier \& Rochex, 1992). Ainsi, l'élève valorise ou dévalorise les savoirs en fonction du sens qu'il leur attribue, et donc se mobilise ou non pour les apprendre. Il est ainsi couramment admis que les apprentissages en milieu difficile amènent de la part de l'enseignant d'EPS une réduction des contenus enseignés ainsi qu'une centration sur les savoir-faire sociaux (Monnier \& Amade-Escot, 2009). Nous pouvons notamment relever une volonté de surajuster des tâches aux caractéristiques des élèves, des difficultés à les impliquer dans de réels enjeux de savoir ou d'apprentissage au-delà de la simple effectuation des tâches, ou encore une baisse des exigences concernant les contenus des programmes visés (Kherroubi \& Rochex, 2004).

Il en est de même concernant leur rapport à la règle et son acceptation. Dans le «football de rue », les joueurs sont partie prenante de la définition des règles et il est rare que des phénomènes de violence se produisent (Cary \& Bergez, 2010). Or, dans le «football de club", les mêmes joueurs qui acceptent de se plier à la règle lorsqu'ils ont eux-mêmes participé à sa définition dans le football improvisé, rejettent violemment la contrainte extérieure (Cary \& Bergez, 2010). Dès lors, il existe un contraste important entre ces deux modalités de pratique du football. Ainsi, pour Long \& Pantaléon (2007), le type de conscience morale du sport auto-organisé est «autonome et ouvert à la négociation» alors que dans le sport institutionnel, cette conscience est «disciplinée et soumise ».

Pour l'enseignant d'EPS, il convient de ne pas négliger les règles constitutives de l'APSA. En effet, l'apprentissage des règles par les élèves amène l'intériorisation de cinq types de règles: les règles du jeu donc, mais également les règles institutionnelles, groupales, d'apprentissage et de sécurité (Méard \& Bertone, 1998). L'élève doit pouvoir se réapproprier les objets d'enseignement retenus par l'enseignant à travers une coconstruction du savoir, telle que préconisée par les nombreux travaux relevant des approches didactiques comparatistes (Amade-Escot, 2007; Schubauer-Leoni, 2008; Sensevy \& Mercier, 2007).

L'important est que les évolutions règlementaires apportées en EPS et en football permettent à l'élève de vivre une réelle expérience de joueur de football, de valoriser leur pratique. Ainsi les conditions de pratique mises en œuvre par l'enseignant, visent à favoriser l'efficacité des apprentissages des élèves dans cette APSA. Aussi, « Apprendre les règles par le jeu permet sans doute un apprentissage plus efficace de celles-ci " (Léziart, 2012). 
En outre, à l'instar de Cary \& Bergez (2010), nous pensons que l'arbitre des rencontres de football se trouve très souvent en "situation de forte vulnérabilité ». Ses décisions sont systématiquement contestées. Or, dans le cadre de l'EPS et des sports-collectifs notamment, les rôles sociaux (arbitre, observateur) semblent être utilisés le plus souvent de manière formelle afin d' "occuper" tous les élèves, et non dans une perspective fonctionnelle en associant les rôles sociaux aux apprentissages. Il convient dès lors de donner les moyens aux élèves d'arbitrer en toute sérénité et de pouvoir progresser dans leur pratique pour ainsi, former des élèves citoyens et acteurs "cultivés, lucides, autonomes" (MEN, 2009) et critiques de l'évolution même du football. Ce regard "critique», cette prise de distance, témoigne d'une intelligence tactique du jeu. Les travaux en didactique des sports collectifs et plus particulièrement en football, montrent l'intérêt d'une prise de distance sur l'action, à travers la construction de "règles d'actions", ce qui a été finalisé par les travaux de recherche relatifs au « débat d'idées » (Gréhaigne \& Godbout, 1998). Le " débat d'idées » permet d'articuler des temps moteurs, par une observation d'une séquence de jeu, avec des temps non-moteurs, par une analyse d'une séquence de jeu. L'enjeu est de conceptualiser l'action pour construire des invariants opératoires et des règles d'action (Gréhaigne, 1992).

En sport collectif, deux notions se trouvent au cœur de l'analyse du jeu: d'une part, celle de configuration de jeu, telle qu'elle s'actualise et prend forme dans le rapport de force en cours, d'autre part, celle de situations potentiellement émergeantes, mais prédictibles à qui sait « lire» le jeu, et dont les joueurs doivent tirer parti (Gréhaigne, Caty \& Marle, 2004). Ces situations "émergeantes" sont également appelées "configurations prototypiques" par Gréhaigne (2007) et permettent une analyse qualitative du jeu en sport collectif. Les enjeux de savoirs associés à la lecture du jeu renvoient à la compétence propre ("Conduire et maitriser un affrontement collectif») et relèvent non seulement de la dimension tactique ("Pertinence et efficacité de l'organisation collective») mais également de l'appropriation des actions collectives signifiantes («Organisation créatrice d'incertitudes par combinaisons d'actions de plusieurs joueurs: circulations de balle, déplacements des joueurs coordonnés, décalages et variation collective du rythme de jeu»). Selon les préconisations institutionnelles, ces objets de savoirs s'actualisent aussi dans la compétence méthodologique («Savoir utiliser différentes démarches pour apprendre »). Les critères d'évaluation qui en résultent sont ainsi liés aux configurations collectives (sur laquelle nous nous centrons dans cet article) et aux conduites motrices individuelles (que nous n'étudions pas dans le cadre de cet article). Ils concernent l'observation spécifique de la circulation du ballon et des joueurs (Chateau, 2004) et l'analyse des séquences de jeu amenant à un tir orienté vers la cible adverse, à partir des indicateurs de jeu collectif suivants: le lieu de la récupération (basse, médiane, haute), la modalité de la récupération (en avant de l'Espace de Jeu Effectif (EJE), au milieu de l'EJE, à la périphérie de l'EJE, en arrière de l'EJE) et le choix de jeu adopté (poursuite du jeu par un tir, une conduite de balle, une passe courte au sol, une passe longue).

L'analyse des configurations de jeu, à partir d'une adaptation des règles du jeu, constitue un outil d'apprentissage et d'évaluation des progrès des élèves relatif aux productions d'actions collectives et sociales telles qu'instituées par les programmes de la discipline. 


\subsection{Le choix d'une approche technologique}

Nous nous inscrivons dans le cadre de l'approche technologique à visée didactique (Bouthier \& Durey, 1994) en prenant appui sur les problèmes de terrain comme objet central d'étude (Bouthier, 2014). Notre recherche a pour but une transformation des pratiques d'intervention, et tente d'apporter des pistes de réflexion quant à l'évaluation des progrès des élèves sur le plan collectif en football.

Nous tenterons de justifier nos choix didactiques par rapport à la pratique sociale de référence et les modalités d'organisation de cette forme de pratique scolaire afin que des élèves issus de milieu "difficile» puissent s'approprier les apprentissages organisationnels, méthodologiques et sociaux (MEN, 2009). Les acquisitions au niveau des savoirs tactiques et méthodologiques d'une classe de Terminale BAC PRO sont évaluées en fonction de l'adaptation de la règle des "fautes collectives " propre au futsal en situation réelle de jeu, et la présence d'élèves co-arbitres. La règle des fautes collectives en futsal peut entraîner un pénalty (à 9 mètres) si une équipe effectue un certain nombre de fautes. Par exemple, pour un match de 8 minutes, si l'équipe commet trois fautes collectives, un pénalty est accordé à l'équipe adverse. Enfin, les « contestations» verbales des joueurs à l'égard des arbitres et des adversaires sont comptabilisées et considérées comme une "faute collective». Nous définissons la " contestation », comme l'action de contester, de ne pas admettre quelque chose, de remettre en cause la décision arbitrale.

\subsection{Problématique et questions de recherche}

5 Face à ces obstacles et aux réticences des enseignants d'EPS à programmer cette activité, notre étude consiste à proposer et à expérimenter une forme de pratique scolaire du football significative pour des élèves "garçons » d'un lycée professionnel situé en Zone Urbaine Sensible (ZUS): le modèle du "futsal», permettant une articulation entre configurations de jeu et construction de la règle en EPS. Nous entendons par « futsal », une forme de pratique scolaire du football, inspirée des règles spécifiques du futsal, en particulier la règle des fautes collectives, mais pratiquée sur terrain extérieur.

Partant de cette problématique, des questions de recherche émergent :

- Quelle forme de pratique scolaire proposer en football afin de faire vivre aux élèves une expérience collective signifiante conciliant leur rapport au savoir et des apprentissages d'actions collectives, méthodologiques et sociaux?

- Considérant que la référence culturelle du futsal et ses règles spécifiques peuvent faire vivre aux élèves une expérience collective signifiante, quels dispositifs l'enseignant d'EPS peut-il concevoir et mettre en œuvre dans cette activité sur la durée d'un cycle?

6 Nous souhaitons ainsi questionner les temporalités propres aux apprentissages des élèves en relation avec celles relatives aux différents dispositifs : a) l'articulation entre temps nécessaire à l'intégration des savoirs tactiques (collectifs); méthodologiques et sociaux et de leur mise en œuvre; $b$ ) la répartition des temps consacrés aux situations d'apprentissage, aux situations de référence et aux débats d'idées, au sein de la leçon et du cycle. 


\section{Méthode}

7 Pour élaborer notre dispositif méthodologique, nous respectons les trois phases caractéristiques de l'approche technologique à visée didactique (Bouthier \& Durey, 1994), à savoir : la conception du projet, sa mise en œuvre et son évaluation.

\subsection{Phase de conception du projet}

8 Cette première phase d'élaboration du projet s'appuie sur les différentes productions théoriques et pratiques afin que ce projet soit cohérent et faisable.

\subsubsection{Une modélisation de l'activité « futsal »}

9 La phase de conception du projet repose sur le modèle d'analyse que nous avons choisi de dénommer « Modélisation futsal» (figure 1).

Figure 1. Modélisation de la forme de pratique scolaire du « futsal »

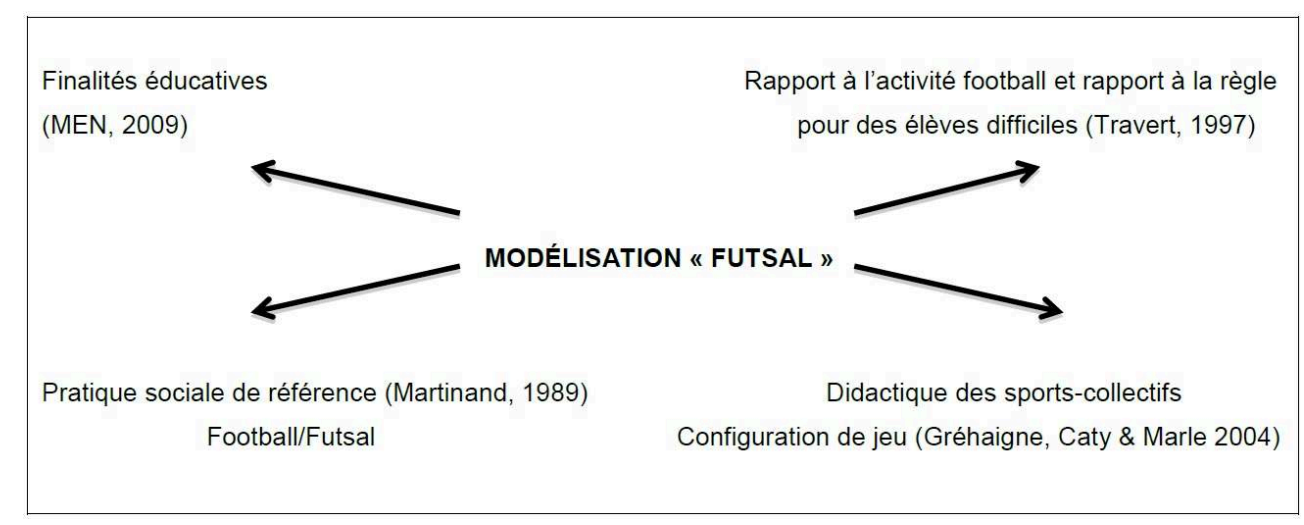

Ce modèle d'analyse du « futsal », schématisé dans la figure 1, tient compte du contexte d'enseignement, des finalités éducatives, des pratiques sociales de référence et des travaux en didactique des sports-collectifs.

\subsubsection{Contexte de l'étude et participants}

11 Le protocole est mis en place dans une classe composée de 18 garçons de Terminale BAC PRO Électrotechnique, issue d'un Lycée Professionnel industriel situé dans une zone urbanisée et socialement défavorisée en Seine-Saint-Denis (93).

Les élèves de cette classe peuvent être considérés comme des élèves "difficiles ", dans la mesure où ils présentent d'importantes difficultés scolaires et peuvent être agités et/ ou perturbateurs.

Ils ont tous un vécu dans la pratique du football (scolaire, UNSS, club, loisir). Ils ne peuvent donc pas être considérés comme des élèves « débutants » dans l'activité. Pour autant, ils reflètent totalement les caractéristiques culturelles développées précédemment dans nos propos introductifs: ils sont orientés principalement sur l'« exploit individuel » en football (Travert, 1997). Ils privilégient systématiquement le dribble et l'action individuelle plutôt que le jeu collectif.

Le protocole est négocié et discuté avec un enseignant de l'équipe pédagogique d'EPS suite à un entretien semi-directif destiné à comprendre l'histoire de l'enseignant. A 
l'issue de cette discussion, nous nous sommes mis d'accord sur l'élaboration du cycle de football, le niveau de classe, le groupe d'élèves pour l'étude de cas, le nombre de séances et la démarche pédagogique. Le rôle de l'enseignant d'EPS renvoie à la conception des situations d'apprentissage, de l'animation de la séance et du suivi des apprentissages des élèves.

\subsection{Phase de mise en œuvre du projet : recueil et traitement des données}

12 Cette deuxième phase de mise en œuvre du projet permet d'expérimenter l'étude, et de tester son efficience.

\subsubsection{Le protocole d'étude}

13 Le protocole d'étude amène les élèves à vivre un cycle de sept leçons de football, activité programmée en classe de Terminale dans l'établissement (cycle 3). La première leçon du cycle (l'évaluation diagnostique) et la dernière leçon (l'évaluation certificative en leçon 7) ne sont pas retenues dans le cadre du dispositif testé. Au regard des objets d'enseignement retenus et étudiés par l'enseignant, le projet de cycle est : «Mettre en cuvre une organisation offensive capable de faire évoluer le rapport de force en sa faveur par une occupation permanente de l'espace de jeu (écartement et étagement), face à une défense qui se replie collectivement pour défendre sa cible ou récupérer la balle" (au regard de la compétence attendue de Niveau 4, MEN, 2009). Cet objectif d'organisation tactique est articulé à un objectif de type méthodologique et social, relatif à l'association de rôles sociaux (observateurs, co-arbitres) à une situation réelle de jeu.

Lors des leçons, les élèves seront regroupés en 3 équipes de 6 joueurs. Les équipes, constituées par l'enseignant d'EPS à partir de la première leçon, sont stables durant le cycle, homogènes entre elles et hétérogènes en leur sain. La structure de leçon « test " reste identique au cours du cycle et respecte les étapes suivantes: un échauffement général et spécifique au football, une situation d'apprentissage en fonction des objets d'enseignement retenus, puis une situation de référence (Gréhaigne \& Cadopi, 1990). La situation de référence a pour fonction, soit de faire émerger des problèmes, soit de valider l'appropriation des savoirs tactiques (collectifs); méthodologiques et sociaux par l'élève. Aussi, à l'instar de Deriaz et Hayoz (2012), nous pensons que la situation de référence doit être aménagée mais authentique, c'est-à-dire respectant les composantes essentielles de l'activité en confrontant l'élève aux problèmes fondamentaux posés par l'APSA pratiquée. Cette notion de situation de référence se différencie de la situation d'apprentissage car elle se focalise plutôt sur l'existence ou non de comportements et non pas sur l'émergence et la construction d'une ou plusieurs règles d'actions en relation avec les pouvoirs moteurs correspondants (Deriaz \& Hayoz, 2012).

La situation de référence articule différents temps :

a. Le premier temps concerne une situation réelle de jeu (8 minutes), dont les règles sont celles $\mathrm{du}$ football avec intégration de la règle des fautes collectives issue du futsal (pénalty à la troisième faute collective ou faute cumulée par équipe). Deux équipes s'affrontent en $6 \mathrm{c} 6$ sur un terrain de $60 \times 45 \mathrm{~m}$, alors que la troisième occupe les rôles sociaux suivants de co-arbitres (deux co-arbitres), d'assistants à la table de marque (chronomètre, score et fautes 
collectives) et d'observateurs (un élève observe les deux co-arbitres et un élève observe les contestations des deux équipes).

b. Le deuxième temps consiste à effectuer un débat d'idées (deux minutes maximum) à l'issue de chaque séquence de jeu. Les trois équipes se regroupent par « collectif ».

c. Le troisième temps réunit les trois «collectifs» autour de l'enseignant afin que chaque collectif puisse débattre et faire part de ses idées au « collectif de la classe » (deux minutes maximum).

d. Le quatrième temps permet d'envisager un bilan des interventions de chacun au sein de la classe (une minute maximum). L'enseignant d'EPS effectue un bilan des interventions de chacun afin de synthétiser les idées et d'orienter les apprentissages.

\subsubsection{Le recueil des données audio et vidéo}

14 L'entretien semi-directif effectué avec l'enseignant d'EPS, d'une durée de 45 minutes, porte sur son parcours d'enseignant, sur ses expériences et sa conception de l'activité football, sur l'organisation prévisionnelle du cycle et enfin, sur sa gestion des élèves et des apprentissages. Le projet est mis en œuvre par un enseignant de l'équipe pédagogique d'EPS autour d'un contrat de recherche positionnant clairement celui-ci comme le seul responsable de l'ensemble du cycle. Ce contrat a été proposé par le chercheur et négocié avec l'enseignant. Le rôle de l'enseignant est dès lors prépondérant dans la mise en œuvre du projet et relève de l'élaboration des situations d'apprentissage, de l'animation de la séance et du guidage des apprentissages des élèves.

En outre, les données recueillies reposent sur l'analyse des séquences de jeu (Gréhaigne, Billard \& Laroche, 1999), à partir d'un enregistrement filmé. La vidéo, cadrée en plan moyen en fonction du déplacement et de l'action des joueurs et des arbitres, suit les déplacements du ballon, l'intervention des arbitres et les réactions des joueurs des deux équipes. Ces séquences peuvent être définies comme les échanges de balles entre les joueurs depuis l'entrée en possession du ballon et jusqu'à sa perte par l'équipe (Gréhaigne, Caty \& Marle, 2004). Ces séquences de jeu permettent d'obtenir des informations importantes comme le nombre d'échanges de balles réussis à l'intérieur de chaque séquence ainsi que le temps de possession durant une séquence avant une interruption du jeu. Ce dernier est très souvent lié au niveau de l'opposition. En effet, plus la séquence de jeu est longue, plus l'équipe démontre qu'elle est capable de gérer différents paramètres du jeu, notamment le jeu adverse en enchaînant différentes unités tactiques.

Toutefois, étant donné que nous ne disposons pas de la direction du jeu et de la position des joueurs, il nous manque deux paramètres importants du jeu. Aussi, il convient d'analyser plus spécifiquement la circulation du ballon et des joueurs (Chateau, 2004). A partir de la notion de phase mère du jeu, définie comme "le lieu d'apparition des déséquilibres à l'origine des attaques, le lieu où l'affrontement pour la récupération du ballon est le plus courant » (Gréhaigne, 2007, p. 93), la méthodologie adoptée consiste avec l'aide de la vidéo à sélectionner et à analyser les séquences de jeu amenant à un tir orienté vers la cible adverse.

Notre analyse s'est effectuée à partir d'une observation croisée de trois critères : le lieu de la récupération (basse, médiane, haute), la modalité de la récupération (en avant de l'Espace de Jeu Effectif (EJE), au milieu de l'EJE, à la périphérie de l'EJE, en arrière de l'EJE) et le choix de jeu adopté (poursuite du jeu par un tir, une conduite de balle, une passe courte au sol, une passe longue). Cette étude des configurations de jeu amenant à 
un tir, devrait nous conduire à identifier des "situations prototypiques", dans le sens où elles représentent un modèle original, archétype d'un modèle qui se reproduit (Gréhaigne, 2007, p. 93). Elle permet d'identifier les conduites motrices collectives à travers l'identification d'indicateurs de jeu collectif.

Enfin, le recueil des données repose sur l'analyse des débats d'idées, en phases 2, 3 et 4, enregistrés et filmés. Toutefois, nous choisissons de nous focaliser uniquement sur l'analyse des configurations de jeu. Nous ne développerons pas l'analyse des débats d'idées dans cet article.

\subsubsection{Le traitement des données}

Le contenu de l'entretien semi-directif effectué avec l'enseignant d'EPS est analysé (Bardin, 1977) puis interprété. Nous regrouperons le contenu et les paroles de l'enseignant en quatre thématiques, afin de prendre en compte les éléments qui nous semblent pertinents pour notre étude. En référence aux fondements de l'approche didactique (Brousseau, 1986), nous chercherons à renseigner les trois instances du " système didactique » : les élèves, le professeur et le savoir. Au regard de notre étude, nous rajouterons une quatrième thématique : la conception de l'activité football.

Le traitement des données issues des séquences de jeu a pour objectif de comparer l'évolution du nombre de fautes collectives, des contestations des joueurs avec l'observation de l'arbitrage. Concernant l'observation des arbitres, nous prenons en compte les critères relatifs aux déplacements, à l'autorité et à la présence, aux gestes et aux explications.

Tableau I. Analyse des séquences de jeu en phase 1 du dispositif

\begin{tabular}{|c|c|c|c|c|c|c|c|c|c|c|c|c|c|c|c|c|}
\hline \multirow{2}{*}{$\begin{array}{c}\text { EJE et } \\
\text { récupératio } \\
n\end{array}$} & \multicolumn{4}{|c|}{$\begin{array}{c}\text { En avant de } \\
\text { I'EJE }\end{array}$} & \multicolumn{4}{|c|}{$\begin{array}{c}\text { Au milieu de } \\
\text { I'EJE }\end{array}$} & \multicolumn{4}{|c|}{$\begin{array}{c}\text { A la périphérie } \\
\text { de l'EJE }\end{array}$} & \multicolumn{4}{|c|}{$\begin{array}{c}\text { En arrière de } \\
\text { I'EJE }\end{array}$} \\
\hline & $j=$ & $\begin{array}{l}\frac{D}{5} \\
\frac{0}{0} \\
0 \\
0 \\
0\end{array}$ & $\begin{array}{l}\bar{D} \\
\infty \\
\Phi \\
\infty \\
\infty \\
\mathbb{0} \\
0\end{array}$ & 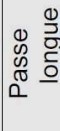 & $i=$ & $\begin{array}{l}\frac{0}{5} \\
\frac{\sigma}{0} \\
0 \\
0\end{array}$ & $\begin{array}{l}\bar{\delta} \\
\infty \\
\Phi \\
\infty \\
0 \\
0 \\
0\end{array}$ & 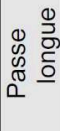 & $i=$ & $\begin{array}{l}\frac{0}{5} \\
\frac{7}{0} \\
0 \\
0\end{array}$ & $\begin{array}{l}\bar{\delta} \\
0 \\
\Phi \\
\Phi \\
0 \\
\sigma \\
0\end{array}$ & 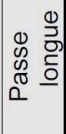 & $j=$ & $\begin{array}{l}\frac{0}{5} \\
\frac{7}{0} \\
0 \\
0\end{array}$ & $\begin{array}{l}\bar{D} \\
\infty \\
0 \\
\infty \\
\infty \\
\tilde{D} \\
0\end{array}$ & $\begin{array}{ll}0 & 0 \\
\infty & 0 \\
\infty & 0 \\
0 & 0 \\
0 & 0\end{array}$ \\
\hline Haute & & & & & & & & & & & & & & & & \\
\hline Médiane & & & & & & & & & & & & & & & & \\
\hline Basse & & & & & & & & & & & & & & & & \\
\hline
\end{tabular}

Les séquences de jeu analysées visent à identifier l'évolution de la « dynamique du jeu » (Gréhaigne, 2007) sur le plan tactique, en attaque et en défense, en fonction de l'objectif de leçon, l'existence de configurations momentanées du jeu se reproduisant durant ces séquences, l'efficacité collective en attaque (nombre de buts) et corrélativement l'efficacité collective en défense (nombre de buts encaissés).

Ainsi, l'analyse et l'identification de configurations de jeu amenant à un tir s'effectuent à partir d'une observation croisée de trois critères (cf. tableau I) : le lieu de la récupération (basse, médiane, haute), la modalité de la récupération (en avant de l'Espace de Jeu Effectif (EJE), au milieu de l'EJE, à la périphérie de l'EJE, en arrière de l'EJE) et le choix de jeu adopté après la récupération du ballon (poursuite du jeu par un tir, une conduite de balle, une passe courte au sol, une passe longue). 


\section{Résultats}

17 Nous présentons la phase d'évaluation du projet en débutant par les résultats concernant l'analyse des séquences $\mathrm{du}$ jeu, puis ceux issus des indicateurs méthodologiques et sociaux.

\subsection{L'analyse des séquences de jeu}

18 L'analyse des séquences de jeu renvoie au premier temps de la situation de référence. Les données chiffrées sont utilisées comme des indicateurs quantitatifs permettant, par l'articulation avec les indicateurs qualitatifs, d'analyser les configurations de jeu et d'approcher au plus près des pratiques des élèves. Les données quantitatives permettent de donner un ordre d'idée des conduites des élèves que les données qualitatives éclairent.

\subsubsection{Les données chiffrées relatives aux évènements du jeu}

19 La figure 2 illustre le temps moyen de possession (durée de la séquence) et le nombre moyen d'échanges de balles réussis à l'intérieur de chaque séquence, définie comme les échanges de balles entre les joueurs depuis l'entrée en possession du ballon et jusqu'à sa perte par l'équipe. Le temps moyen de possession est compris entre 7 et 10 secondes, tandis que le nombre moyen d'échanges réussis est compris entre 2 et 3 . Comme révélé par la figure 2, cette durée et ce nombre d'échanges sont relativement constants au cours du cycle, mais présentent tout de même une légère augmentation en leçon 4 (10 secondes de temps moyen de possession de balle).

A ce titre, l'objectif de la leçon 4 était de «S'organiser collectivement en attaque en occupant tout l'espace de jeu face à une défense placée ». L'objectif collectif était ainsi axé sur la conservation $\mathrm{du}$ ballon et peut expliquer le temps moyen de possession plus conséquent et le nombre d'échanges réussis. En outre, selon Gréhaigne, Billard et Laroche (1999), " plus la séquence de jeu est longue plus l'équipe prouve qu'elle est à même de gérer différents paramètres du jeu, et en particulier le jeu adverse en enchaînant diverses unités tactiques ». Nous pouvons notamment prendre l'exemple de l'équipe C qui a un temps moyen de possession de 12 secondes. Ce temps moyen de possession est supérieur aux autres équipes $A$ et $B$ et se concrétise également par une efficacité collective en attaque, eu égard aux deux victoires de l'équipe $C$ lors de cette leçon (2-1 contre l'équipe $A$ et 2-0 contre l'équipe B). 
Figure 2. Evolution du temps moyen de possession et du nombre moyen d'échanges réussis par séquence de jeu analysée au cours du cycle (moyenne pour l'ensemble des équipes)

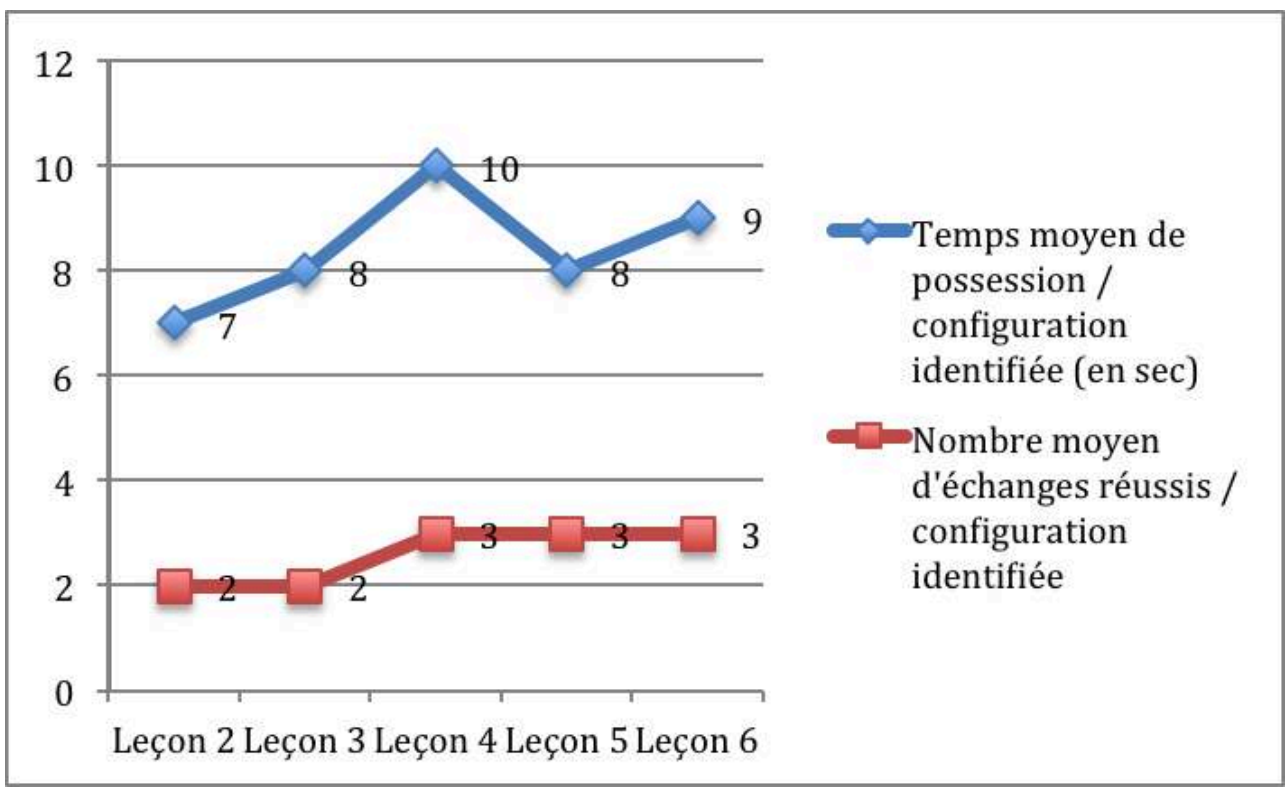

\subsubsection{L'efficacité collective en attaque et en défense}

Comme illustré par la figure 3, les courbes et les évolutions du nombre moyen de tirs et du nombre moyen de buts subissent la même irrégularité en fonction des leçons du cycle.

Figure 3. Evolution du nombre moyen de tirs et du nombre moyen de buts marqués au cours du cycle (moyenne pour l'ensemble des équipes)

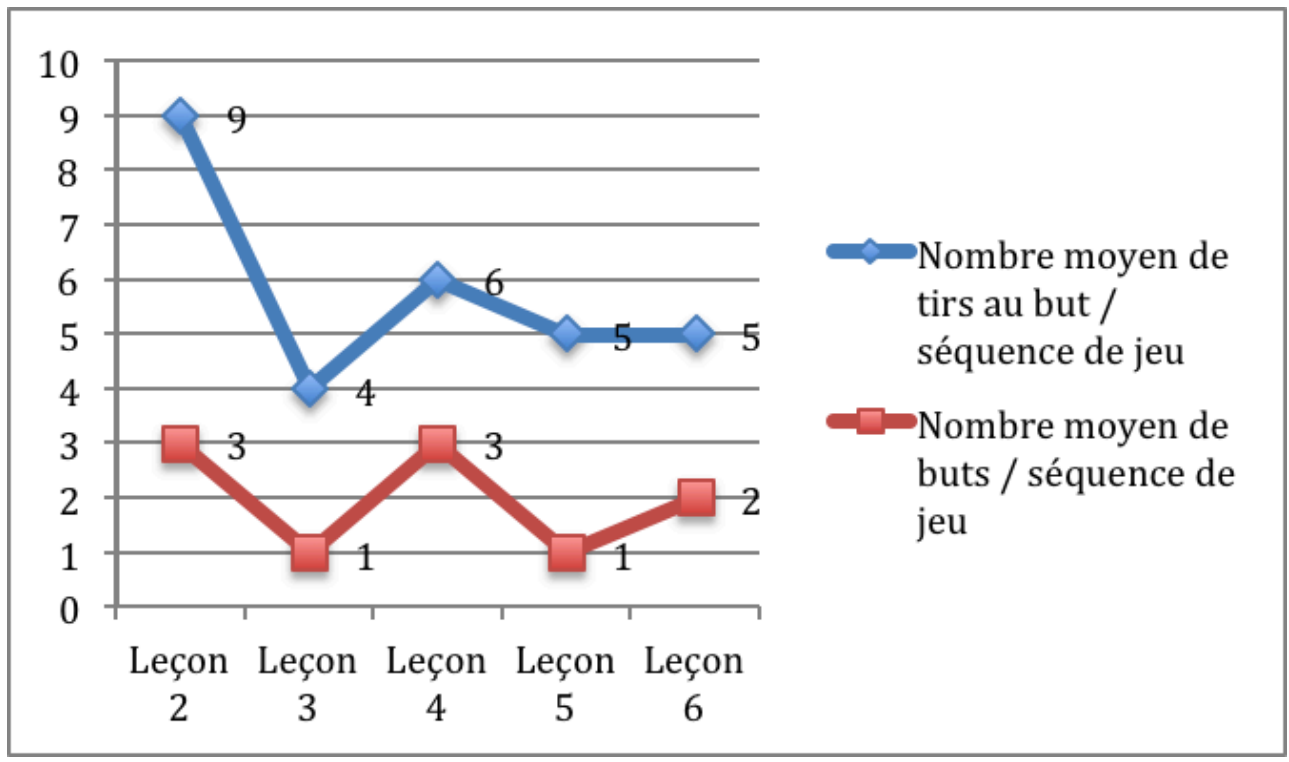

Les résultats montrent un nombre important de tirs et de buts marqués en leçon 2 ( 9 tirs et 3 buts marqués), 4 ( 6 tirs et 3 buts marqués) et 6 ( 5 tirs et 2 buts marqués) et témoignent d'une certaine « efficacité collective en attaque ». Ils peuvent être corrélés aux objectifs des leçons 2, 4 et 6, centrés sur des contenus et des apprentissages "offensifs", sur des aspects de l'organisation collective en attaque. A contrario, les 
leçons 3 et 5 présentent des résultats et une "efficacité collective en attaque " plus relative (4 et 5 tirs pour 1 but marqué en moyenne). Ces leçons sont centrées sur des objectifs « défensifs ", dont l'organisation collective en défense et attestent de la même manière d'une relative « efficacité collective en défense. Toutefois, le nombre moyen de tirs au but présente une évolution décroissante durant le cycle ( 9 tirs en leçon 2 et 5 tirs en leçon 6). Cette diminution atteste d'un rééquilibrage du rapport de force et des organisations collectives des équipes, en attaque et en défense. Il semblerait donc qu'il soit plus facile de progresser en défense qu'en attaque. De plus, les objets d'enseignement retenus par l'enseignant peuvent expliquer cette relation entre objectif de leçon et efficacité collective. A titre d'exemple, le choix de l'enseignant de se centrer sur «S'organiser en défense de zone et conserver le bloc défensif: près et loin du but » en leçon 5 , amène les élèves à se centrer sur la protection de leur but et donc sur l'efficacité collective en défense ( 1 but marqué en moyenne).

\subsubsection{Les configurations du jeu}

Le tableau II relève le nombre de configurations de jeu amenant à un tir à partir de l'analyse vidéo de 70 séquences de jeu sélectionnées au cours du cycle. Comme révélé par le tableau III, sur les 70 séquences de jeu retenues, cinq configurations apparaissent et se sont reproduites plus fréquemment au cours du cycle.

Tableau II. Comptabilisation des configurations de jeu amenant à un tir

\begin{tabular}{|c|c|c|c|c|c|c|c|c|c|c|c|c|c|c|c|c|}
\hline $\begin{array}{c}\text { EJE et } \\
\text { récupération }\end{array}$ & \multicolumn{4}{|c|}{ En avant de l'EJE } & \multicolumn{4}{|c|}{ Au milieu de l'EJE } & \multicolumn{4}{|c|}{$\begin{array}{c}\text { A la périphérie de } \\
\text { l'EJE }\end{array}$} & \multicolumn{4}{|c|}{$\begin{array}{c}\text { En arrière de } \\
\text { l'EJE }\end{array}$} \\
\hline $\begin{array}{c}\text { Choix de jeu } \\
\text { adopté }\end{array}$ & 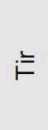 & $\begin{array}{l}\stackrel{0}{5} \\
\frac{7}{0} \\
\text { 어 }\end{array}$ & $\begin{array}{l}\overline{0} \\
\infty \\
\Phi \\
\mathscr{D} \\
\mathbb{D} \\
0 \\
0\end{array}$ & 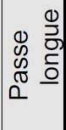 & $i=$ & $\begin{array}{l}\stackrel{0}{5} \\
\frac{7}{0} \\
0 \\
0 \\
0\end{array}$ & 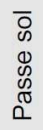 & 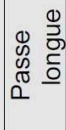 & $i=$ & $\begin{array}{l}\frac{0}{7} \\
\frac{1}{0} \\
0 \\
0\end{array}$ & $\begin{array}{l}\bar{\delta} \\
\infty \\
\infty \\
\infty \\
\infty \\
\infty \\
0 \\
0\end{array}$ & 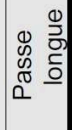 & $\vdots \vDash$ & 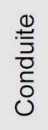 & $\begin{array}{l}\overline{0} \\
\infty \\
\otimes \\
\otimes \\
\Phi \\
\tilde{D} \\
\alpha\end{array}$ & 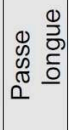 \\
\hline Haute & 3 & 7 & 4 & & & & 2 & & & 3 & 1 & & & 3 & & \\
\hline Médiane & & & 3 & & 1 & 2 & 9 & & & 3 & 6 & & & 1 & 7 & \\
\hline Basse & & & & & & & & & & 1 & 2 & & & 6 & 3 & 3 \\
\hline
\end{tabular}

La récupération haute en avant de l'EJE suivie d'une conduite de balle par le joueur est apparue 7 fois (soit $10 \%$ des configurations de jeu identifiées). Cette configuration peut être la conséquence d'un pressing des attaquants ayant la volonté de progresser rapidement vers la cible adverse, ce qui correspond à l'objet d'enseignement retenu par l'enseignant en leçon 2.

La récupération médiane au milieu de l'EJE suivie d'une passe courte au sol par le joueur s'est reproduite 9 fois (soit $13 \%$ des configurations de jeu identifiées). Cette configuration est le plus souvent la conséquence d'un pressing des défenseurs. L'objectif est alors de "s'organiser collectivement en défense et maintenir l'écart entre les lignes » (objet d'enseignement de la leçon 3).

La récupération médiane à la périphérie de l'EJE suivie d'une passe courte au sol par le joueur a été observée 6 fois (soit $9 \%$ des configurations de jeu identifiées). Cette autre configuration survient le plus souvent à la suite d'une perte de balle d'un défenseur. Cette récupération à la périphérie du jeu semble liée à la volonté de l'équipe de " contourner et déséquilibrer » la défense adverse (objet d'enseignement de la leçon 6).

La récupération médiane en arrière de l'EJE suivie d'une passe courte au sol par le joueur s'est présentée 7 fois (soit $10 \%$ des configurations de jeu identifiées). Cette configuration peut s'expliquer par la difficulté pour l'attaque à progresser vers la cible face à une défense regroupée dans l'axe (objet d'enseignement de la leçon 3). 
La récupération basse en arrière de l'EJE suivie d'une conduite de balle par le joueur a été identifiée 6 fois (soit $9 \%$ des configurations de jeu identifiées). Cette configuration est la conséquence de la difficulté de l'attaque à pénétrer dans le dispositif défensif avec une récupération basse à l'arrière de l'espace de jeu effectif puis jeu de transition en déviation. Cette récupération basse témoigne d'une " organisation en bloc défensif près de son propre but", objet d'enseignement retenu par l'enseignant en leçon 5. Cette récupération basse semble renforcée par la limitation des fautes qui est une règle empruntée au futsal, ce qui amène les élèves à adapter leur intervention défensive sans faire de faute.

Le tableau III nous permet d'identifier cinq configurations prototypiques, dans le sens où elles représentent un modèle qui se reproduit au cours du cycle.

Tableau III. Identification de cinq configurations prototypiques à partir de la sélection et de l'analyse vidéo de 70 séquences de jeu amenant à un tir, durant le cycle

\begin{tabular}{|c|c|c|c|c|c|c|c|c|c|c|c|c|c|c|c|c|}
\hline \multirow{2}{*}{$\begin{array}{c}\text { EJE et } \\
\text { récupération } \\
\text { Choix de jeu } \\
\text { adopté }\end{array}$} & \multicolumn{4}{|c|}{ En avant de l'EJE } & \multicolumn{4}{|c|}{$\begin{array}{l}\text { Au milieu de } \\
\text { l'EJE }\end{array}$} & \multicolumn{4}{|c|}{$\begin{array}{c}\text { A la périphérie } \\
\text { de l'EJE }\end{array}$} & \multicolumn{4}{|c|}{$\begin{array}{l}\text { En arrière de } \\
\text { I'EJE }\end{array}$} \\
\hline & 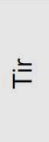 & $\begin{array}{l}\frac{0}{5} \\
\frac{7}{0} \\
\frac{0}{0} \\
0\end{array}$ & $\begin{array}{l}\bar{D} \\
i \\
0 \\
0 \\
0 \\
0 \\
0 \\
0\end{array}$ & $\begin{array}{ll}0 & 0 \\
0 & 0 \\
w & 0 \\
0 & 0 \\
0 & 0\end{array}$ & $i$ & $\begin{array}{l}\frac{0}{5} \\
\frac{3}{0} \\
0 \\
0 \\
0\end{array}$ & $\begin{array}{l}\overline{0} \\
\infty \\
0 \\
\infty \\
0 \\
0 \\
0\end{array}$ & \begin{tabular}{ll} 
& 0 \\
$\infty$ & 0 \\
$\infty$ & 0 \\
0 & 0 \\
\hdashline & 0 \\
0 & 0
\end{tabular} & $\equiv$ & $\begin{array}{l}0 \\
\stackrel{5}{5} \\
\frac{0}{0} \\
0 \\
0\end{array}$ & $\begin{array}{l}\bar{D} \\
i \\
0 \\
0 \\
0 \\
0 \\
0\end{array}$ & 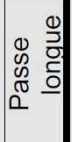 & 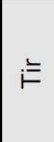 & $\begin{array}{l}\frac{9}{5} \\
\frac{3}{0} \\
0 \\
0 \\
0\end{array}$ & $\begin{array}{l}\bar{D} \\
\infty \\
0 \\
\infty \\
0 \\
\sigma \\
0\end{array}$ & $\begin{array}{ll}0 & 0 \\
\infty & \vec{z} \\
\infty & 0 \\
0 & 0 \\
\sigma & 0 \\
0 & 0\end{array}$ \\
\hline Haute & & $x$ & & & & & & & & & & & & & & \\
\hline Médiane & & & & & & & $x$ & & & & $x$ & & & & $x$ & \\
\hline Basse & & & & & & & & & & & & & & $X$ & & \\
\hline
\end{tabular}

\subsection{Les indicateurs méthodologiques et sociaux}

Nous présentons les résultats concernant les indicateurs méthodologiques et sociaux, qui s'articulent avec les données précédentes relatives à l'analyse des séquences de jeu.

\subsubsection{Les données relatives aux fautes collectives et aux contestations}

Comme révélé par la figure 4 , les résultats montrent une diminution importante du nombre de contestations des joueurs au cours du cycle ( 6 en moyenne en leçon 2 et 1 en moyenne en leçon 6). Parallèlement, le nombre de fautes collectives par séquence de jeu diminue également sensiblement durant le cycle (3 en leçon 2 et 1 en leçon 6). 
Figure 4. Evolution du nombre moyen de fautes collectives et du nombre moyen de contestations des joueurs au cours du cycle (moyenne pour l'ensemble des équipes)

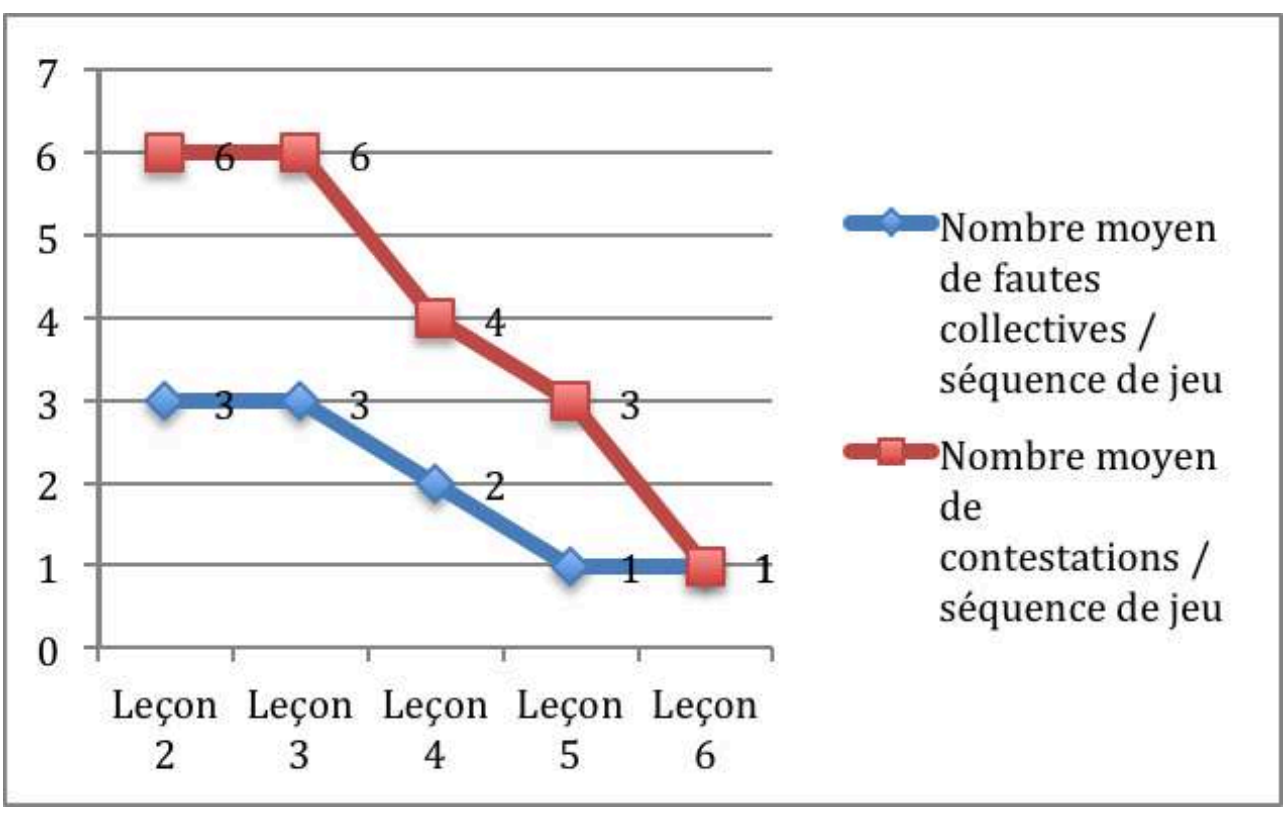

\subsubsection{L'observation des co-arbitres}

A partir de la fiche d'observation des deux élèves co-arbitres lors des séquences de jeu, les élèves observateurs ont évalué les arbitres à partir de critères d'observation relatifs aux «déplacements", à "l'autorité et la présence», ainsi qu'aux "gestes et aux explications ». Majoritairement, au début du cycle, les élèves ont observé des arbitres " assez loin de l'action ", se déplaçant pour certains mais sans anticiper l'action à venir. Ils ont pu observer des arbitres « hésitant dans leurs prises de décision», subissant les "contestations" des joueurs. Cette hésitation lors des premières leçons peut s'expliquer par une méconnaissance pour certains du règlement et un "manque d'explication et de communication» des arbitres avec les joueurs. En fin de cycle, les élèves observateurs ont relevé des arbitres bien plus "mobiles ", "sifflant plus » et «aidant les joueurs par leurs gestes ». Cette évolution positive des élèves dans le rôle de co-arbitre s'explique également par une diminution significative des contestations des joueurs (cf. figure 4). Ainsi, les contestations des décisions de l'arbitre ( Les arbitres ne sifflent pas trop les fautes ") et de sa connaissance du règlement ("J'ai vu plusieurs mains et l'arbitre n'a pas sifflé»), ont diminué sensiblement au profit d'un nombre de fautes collectives limitées et relatif à des fautes de jeu (contact ou tacle par exemple).

\section{Discussion}

Notre ambition était d'étudier l'impact d'une forme de pratique scolaire du football dérivée $\mathrm{du}$ futsal sur l'activité d'élèves issus de milieu difficile et sur leurs apprentissages relatifs aux productions d'actions collectives (savoirs tactiques) ainsi que méthodologiques et sociales. La discussion des résultats vise à mettre en évidence un certain nombre de connaissances utiles à la décision dans un acte d'enseignement (Bouthier \& Durey, 1994). 


\subsection{La relation entre objectif de leçon et efficacité collective} aspect offensif ou un aspect défensif d'un point de vue de l'organisation collective. En effet, l'analyse de l'efficacité collective des équipes montre que le nombre de tirs et de buts marqués est plus important pour une leçon centrée sur un objectif " offensif »; et de la même manière le nombre de buts encaissés est faible lors de leçons axées sur des objectifs « défensifs ». Nos résultats ne nous permettent pas de prouver avec certitude que ce sont les objectifs de leçon et les objets d'enseignement travaillés qui ont entraîné cette corrélation, mais nous pensons que cette tendance peut toutefois être envisagée. Partant de cette interprétation, notre étude permet de mettre en avant un « réinvestissement » des savoirs tactiques collectifs par les élèves lors de la situation de référence. Les résultats de l'entretien ante réalisé avec l'enseignant semblent confirmer cette tendance, puisque selon l'enseignant il convient d'axer le projet de cycle sur « la dimension collective et l'aspect tactique ». En outre, les données issues de l'analyse des débats d'idées nous permettent également de rendre compte de ce réinvestissement des apprentissages résultant des échanges entre les élèves.

Néanmoins, notre approche présente certaines limites au regard de l'adaptation nécessaire des systèmes de jeu par rapport à l'adversaire. En effet, le rééquilibrage du rapport de forces attaque/défense et donc de l'efficacité collective en attaque et en défense, opéré en leçon 6 , témoigne d'une certaine application et non d'une adaptation des configurations de jeu. Avec Caty, Meunier et Gréhaigne (2007), nous pensons qu'il convient de concevoir l'organisation du jeu comme une totalité, ce qui revient à penser l'attaque et la défense ensemble et dans le même instant. Les prescriptions institutionnelles en matière d'évaluation de l'efficacité collective de l'activité football au Baccalauréat Professionnel (MEN, 2009) vont également dans ce sens puisqu'elles préconisent d'envisager à chaque leçon, de manière systémique et imbriquée, l'organisation collective du jeu en attaque et en défense.

\subsection{Les configurations prototypiques au service de l'analyse du jeu}

Nos résultats nous ont permis d'identifier cinq configurations prototypiques à partir de l'analyse qualitative du jeu en sport collectif concernant 70 séquences de jeu amenant à un tir. Notre étude confirme les résultats observés par Gréhaigne (2007) reposant sur l'étude de 98 séquences de jeu. Toutefois, des disparités ont été observées et peuvent s'expliquer par la différence de niveau des élèves dans l'activité football. En effet, nos élèves ne sont pas "débutants" dans l'activité, puisqu'ils ont tous un vécu dans la pratique du football (scolaire, UNSS, club, loisir). De plus, notre étude se centre sur une pratique inspirée de la règle du jeu du futsal : les fautes collectives.

Certaines configurations de jeu identifiées nous permettent de caractériser le niveau de nos élèves à l'interface entre le Niveau 3 et le Niveau 4 de la Compétence attendue (MEN, 2009). Ainsi, «La récupération médiane au milieu de l'EJE suivie d'une passe courte au sol par le joueur » ou «La récupération médiane en arrière de l'EJE suivie d'une passe courte au sol par le joueur ", témoignent d'une configuration de jeu initiale quand l'espace de jeu effectif s'est stabilisé dans le demi-terrain offensif. Ces configurations peuvent être la conséquence de la difficulté de l'attaque à pénétrer dans le dispositif défensif avec une récupération basse ou médiane, au milieu ou à l'arrière de l'espace de jeu effectif, puis un jeu de transition en déviation sous forme de passes courtes au sol, puis tir au but. Le

eJRIEPS, 35 | 2015 
temps de possession augmente, même si en jeu nos résultats montrent que le nombre d'échanges réussis dépasse rarement deux ou trois échanges.

L'identification de ces configurations prototypiques permet d'évaluer un niveau de compétence attendue et qualifier les transformations des actions collectives des élèves telles qu'elles sont identifiées à travers les configurations collectives de jeu sur la temporalité d'un cycle. Ainsi, la configuration de jeu observée en début de cycle (leçon 2) : "La récupération médiane au milieu de l'EJE suivie d'une passe courte au sol par le joueur ", correspond à un niveau 3 de compétence en cours d'acquisition: une "organisation offensive simple, fondée sur l'action individuelle du porteur de balle, début d'échanges entre deux partenaires dans l'axe du but, pour accéder à l'espace de marque ». En fin de cycle (leçon 5), la configuration de jeu : "La récupération médiane en arrière de l'EJE suivie d'une passe courte au sol par le joueur » répond à un niveau 4 de compétence attendue : une «organisation offensive liée à des enchaînements d'actions privilégiant l'utilisation alternative des couloirs latéraux et du couloir central. Echanges qui créent le déséquilibre». L'évolution des configurations de jeu identifiées, analysées et donc d'un niveau de compétence attendue, atteste de transformations du niveau de jeu collectif des élèves au cours du cycle. Aussi, ces résultats peuvent apporter une aide aux pratiques des enseignants, notamment dans l'optique d'évaluer l'efficacité collective des élèves en football.

Après ce travail d'identification et de sélection de situations prototypiques, se pose maintenant le problème de leur utilisation par les élèves. Aussi, nous postulons que l'utilisation de l'outil vidéo en sport collectif est susceptible de favoriser leur réutilisation en situation de jeu (Berchebru, Meunier \& Gréhaigne, 2009). Partant de cette idée, une étude future pourrait se centrer sur le visionnage par les élèves des configurations de jeu choisies avec pour objectif de viser une adaptation des organisations collectives retenues en fonction du rapport de force adverse. Cette future étude consisterait à un prolongement du débat d'idées qui intégrerait l'utilisation d'outils vidéo, ce qui renvoie à l'acquisition de compétences scolaires.

\subsection{L'impact des règles du jeu sur les productions d'actions collectives, sur les apprentissages méthodologiques et sociaux}

30 Les résultats observés montrent que l'instauration de la règle des fautes collectives a entraîné une diminution sensible des contestations des joueurs lors des séquences de jeu. De plus, cette construction collective de la réponse par rapport au rôle d'arbitre, a permis d'observer une amélioration certaine de l'occupation des rôles sociaux, contribuant ainsi à de réels apprentissages au niveau méthodologique : au niveau de leurs déplacements, de leur autorité et de leur présence, et de leur communication avec les joueurs. Nous pouvons également faire l'hypothèse d'une influence directe de l'instauration de la règle des fautes collectives sur les apprentissages moteurs des élèves au niveau de la fluidité du jeu en attaque et au niveau de l'intervention du défenseur. En effet, l'enseignant s'est focalisé sur cet objet d'apprentissage au cours du cycle. De plus, selon les résultats de l'entretien ante effectué avec l'enseignant, la relation professeur/élève doit être basée "sur un code commun sur le règlement du jeu, pour éviter tout conflit et toute frustration de l'élève ». 


\subsection{L'enseignement du football en milieu difficile : le modèle du «futsal » en EPS}

31 L'ambition de notre travail était d'actualiser une forme de pratique scolaire du football adaptée à notre public, des élèves « difficiles ». Nous pensons à cet égard que le modèle $\mathrm{du}$ « futsal », tant dans l'adaptation des règles du jeu que dans l'alternance de temps de pratique et de temps de débat d'idées, permet de prendre en compte le rapport au sens construit par des élèves issus de milieu difficile. En effet, la valorisation du collectif, la limitation des fautes et des contestations, l'implication des élèves dans les rôles sociaux, la co-construction des savoirs moteurs collectifs, méthodologiques et sociaux, ou encore l'usage de l'outil vidéo sont autant d'arguments en faveur de cette forme de pratique scolaire en EPS.

Enfin, il convient de rester attentif au contexte culturel et social de l'élève, puisque " faire vivre une expérience culturellement signifiante aux élèves en EPS est une ambition complexe, car quoi qu'il en soit la seule mise en référence culturelle effectuée par l'enseignant n'est pas garante du sens que les élèves vont donner à leur pratique» (Mascret, 2009). L'enseignant joue ainsi le rôle de "guide » et non de "prescripteur » de l'activité de l'élève. Aussi, dans la perspective de la théorie de l'action conjointe en didactique (Schubauer-Leoni \& Leutenegger, 2005; Sensevy \& Mercier, 2007), les élèves et l'enseignant co-construisent une référence commune, elle-même articulée aux préconstruits socio-culturels.

Dans le cadre de notre forme de pratique scolaire, l'expérience aura du sens pour l'élève s'il s'intéresse à l'action collective, s'il y voit un intérêt personnel, si les élèves sentent qu'ils dépendent les uns des autres et adoptent des comportements qui le permettent, et enfin si les règles mises en place amènent une plus value dans leur pratique. L'important est que les évolutions règlementaires apportées en EPS et en football amènent l'élève à vivre une réelle expérience de joueur de football, et que les conditions de pratique, mises en œuvre par l'enseignant, lui permettent de devenir plus efficace dans cette APSA.

\section{Conclusion}

L'objectif de notre étude était de présenter ses apports et son impact du point de vue de sa " visée transformative » (Schwartz, 1997). Elle décrit l'apport pour les élèves et pour l'enseignant ayant participé à cette étude. Pour des élèves difficiles, une coconstruction des savoirs et une réflexion collective à travers des débats d'idées, permettent de produire des actions collectives signifiantes, des apprentissages méthodologiques et sociaux dans un sport collectif comme le football. A cet égard, l'instauration de la règle des fautes collectives et l'observation des contestations des joueurs peuvent faciliter l'implication de l'élève dans le rôle d'arbitre, mais également les acquisitions motrices de type collectif ou individuel dans le jeu. L'action motrice d'un élève qui maîtrise ses déplacements et son engagement dans l'action de reconquête de balle en est un exemple. Les résultats de notre étude peuvent amener à une réflexion sur la formalisation de savoirs "pour" et "par" l'arbitre ou l'observateur à travers l'intégration des rôles sociaux (arbitres, observateurs) dans la pratique. L'enjeu réel de l'élève observateur et de l'élève arbitre n'est pas tant d'occuper les élèves de manière formelle, mais bel et bien de les impliquer dans 
l'analyse des réponses en jeu afin de faire de ce moment un temps d'apprentissage authentique.

L'identification de configurations prototypiques nous permet d'analyser la qualité du jeu des élèves et ainsi caractériser leurs progrès et leur niveau de compétence. Parallèlement, la prise en compte de l'efficacité collective en attaque et en défense permet d'envisager l'utilisation et le réinvestissement d'objectifs de leçon centrés sur les aspects défensifs ou offensifs.

Nos résultats peuvent également influencer l'intervention de l'enseignant d'EPS lors de l'élaboration d'une forme de pratique scolaire, en sport collectif notamment. Ainsi, l'implication de l'élève dans les tâches d'arbitre ou d'observateur nécessite de la part de l'enseignant la recherche de sens, notamment pour des élèves issus de milieux difficiles, puisque «le sens n'est pas un objet que l'on enseigne, mais un rapport que le sujet construit» (Rochex, 1996).

Toutefois, notre recherche présente certaines limites. En effet, nos résultats, centrés sur des élèves d'une classe et d'un cycle de football donnés, ne nous permettent pas de certifier certains éléments apportés par notre étude, notamment eu égard au réinvestissement d'objectifs "offensifs" ou "défensifs" en fonction de la leçon étudiée. Ces limites nous amènent à penser que l'utilisation de configurations de jeu par les élèves doit être pensée comme une totalité et ainsi, l'enseignant doit préconiser dans une même leçon des objectifs sur le plan offensif et défensif (Caty, Meunier \& Gréhaigne, 2007). Il convient enfin de nuancer la généralisation des résultats à l'ensemble des élèves issus de milieux difficiles au regard du contexte de cette étude, qui concerne une classe de Terminale BAC PRO, dont les élèves ont pour perspective proche le baccalauréat, ce qui atteste d'une certaine réussite de leur parcours. Pour autant, en mettant la focale sur la production d'actions collectives organisées des élèves dans la mise en œuvre d'une forme de pratique scolaire, l'analyse de configurations de jeu renseigne les manières dont ils peuvent atteindre un niveau de compétence et viser de réels apprentissages. Plus largement, en intégrant les dimensions motrices et méthodologiques des programmes d'EPS, la forme de pratique scolaire du «futsal » peut contribuer à la mise en place d'une approche plus positive du football en EPS tout en respectant le rapport au sens construit par des élèves difficiles. Finalement, notre étude mériterait d'être prolongée et ainsi élargie à plusieurs classes, sur plusieurs cycles afin de confirmer ou d'infirmer nos hypothèses de recherche et les résultats présentés, analysés et discutés dans le cadre de cet article.

\section{BIBLIOGRAPHIE}

Amade-Escot, C. (2007). Les savoirs, au cœur du didactique. In C. Amade-Escot (Ed.), Le Didactique (pp. 11-30). Paris : Revue EPS, collection « Pour l'action ».

Bardin, L. (1977). L'analyse de contenu. Paris : PUF.

Beaud, S. (2003). 80 \% au bac... et après ? Les enfants de la démocratisation scolaire. Paris : La

Découverte.

eJRIEPS, 35 | 2015 
Beaud, S., \& Guimard, P. (2014). L'entrée dans le métier et l'ouverture internationale. Dans S. Beaud, \& P. Guimard (Eds.). Affreux, riches et méchants? Un autre regard sur les bleus (pp. 17-43). Paris : La Découverte.

Becker, A. (2014). Le foot à l'école. Simple brouille ou divorce consommé ? Revue Contre Pied, HorsSérie n 9, mai, 4-6.

Berchebru, M., Meunier, J.-N., \& Gréhaigne J.-F. (2009). L'utilisation de films vidéo illustrant des configurations prototypiques du jeu et de débats d'idées dans la didactique du football en milieu scolaire. In J.-F. Gréhaigne, (Ed.) Autour du temps. Espaces, apprentissages, projets dans les sports collectifs (pp. 103-126). Besançon : Presses de l'Université de Franche-Comté.

Bouthier, D. (2014). Approche techno-didactique de l'influence des pratiques sociales sur l'intervention éducative en EPS et en sport. Conférence présentée à la $8^{\text {ème }}$ Biennale de l'ARIS « Temps, temporalités et intervention en EPS et en sport ». Genève. 02-04 Juillet 2014.

Bouthier, D., \& Durey, A. (1994). Technologie des APS. Impulsions, 1, 95-124.

Brousseau, G. (1986). Fondements et méthodes de la didactique des mathématiques. Recherches en didactique des mathématiques, 7/2, 33-115

Caillé, A. (2008). Au-delà de l'intérêt, Revue du Mauss semestrielle, 31, 95-115.

Cary, P., \& Bergez, J.L. (2010). Violence, identité et reconnaissance dans le football en milieu populaire, Sociologies [En ligne], Théories et recherches, mis en ligne le 04 février 2010. URL : http://sociologies.revues.org/3022.

Caty, D., Meunier, J.N., \& Gréhaigne, J.F. (2007). Modélisations des attaques réussies pour progresser dans les sports collectifs en EPS. Spirales, 40, 105-116.

Charlot, B., Bautier, E., \& Rochex, J-Y. (1992). Ecole et savoir dans les banlieues... et ailleurs. Paris : Armand Colin.

Chateau, L. (2004). Analyse sémiotique des configurations prototypiques offensives chez les débutants en football. Mémoire de DEA en Science du langage, didactique, sémiotique. Université de FrancheComté.

Deriaz, D., \& Hayoz, C. (2012). De l'intérêt de l'image et de la participation des élèves à la construction des règles du jeu en gymnastique : une expérience au saut de cheval ! eJRIEPS, 25, 80-102.

Dubet, F., \& Duru-Bellat, M. (2000). L'hypocrisie scolaire : pour un collège enfin démocratique. Editions du Seuil.

Frantz, P. (1975). Le Football. Mulhouse : L’Alsace.

Gréhaigne, J.F. (1992). L'organisation du jeu en football. Paris : ACTIO.

Gréhaigne, J.F. (Ed.). (2007). Configurations du jeu, débat d'idées et apprentissage des sports collectifs. Besançon : Presses de l'Université de Franche-Comté.

Gréhaigne, J.F., Billard, M., \& Laroche, J.Y. (1999). L'enseignement des jeux sportifs collectifs à l'école. Conception, construction, évaluation. Bruxelles : De Boeck.

Gréhaigne, J. F., \& Cadopi, M. (1990). Apprendre en éducation physique. Dans Éducation physique et didactique des APS (pp. 17-24). Paris : AEEPS.

Gréhaigne, J.F., Caty, D., \& Marle, P. (2004). L'apport de la notion de configuration du jeu à la didactique des sports collectifs. In G. Carlier (Ed.), Si l'on parlait du plaisir d'enseigner l'éducation physique (pp. 167-179). Montpellier : AFRAPS. 
Gréhaigne, J.F., \& Godbout, P. (1998). Observation, critical thinking and transformation : Three key elements for a constructivist perspective of the learning process in team sports. In R. Feingold, R. Rees, G. Barrette, L. Fiorentino, S. Virgilio, \& E. Kowalski (Eds.), Education for life (pp. 109-118). New York : Adelphi University.

Kherroubi, M., \& Rochex, J.Y. (2004). La recherche en éducation et les ZEP en France. 2. Apprentissages et exercice professionnel en ZEP : résultats, analyses, interprétations. Revue française de pédagogie 146, 115-190.

Kievits-Boucher, R. (2006). Le futsal (Version originale du futebol de saläo), éd. Française éditée et publiée par l'UNCFs avec autorisation de la LFFS.

Lepoutre, D. (1997). Les compétitions honorifiques. Dans D. Lepoutre (Ed.). Cour de banlieue. Codes, rites et langages (pp. 398-403). Paris : Éditions Odile Jacob.

Léziart, Y. (2012). La règle sportive, reflet de l'intelligence humaine en action : approche anthropologique. Dans Y. Léziart, G. Cabagno, M. Loquet, J. Trohe (Eds.), La règle sportive (pp. 17-41). Bordeaux : Presses universitaires de Bordeaux.

Long, T., \& Pantaléon, N. (2007). Étude des relations entre conscience réglementaire et contextes de pratique sportive auprès d'adolescents sportifs, Staps, 75, 43-58.

Martinand, J-L. (1989). Pratiques de référence, transposition didactique et savoirs professionnels en sciences et techniques. Les sciences de l'éducation, pour l'ère nouvelle, 1-2, 23-35.

Mascret, N. (2009). Référence culturelle et formation des enseignants d'Education Physique et Sportive, Travail et Formation en Education, 3, [En ligne], mis en ligne le 18 décembre 2009. URL : http://tfe.revues.org/index870.html.

Mascret, N., \& Dhellemmes, R. (2011). Culture sportive et culture scolaire des APSA. Dans M. Travert, et N. Mascret (Eds.) La culture sportive (pp. 99-115). Paris : Éditions EP\&S.

Mauny, C. \& Gibout, C. (2008). Le football "sauvage" : d'une autre pratique à une pratique autrement, Science et motricité, 62 (1), 53-61.

Méard, J.A. \& Bertone, S. (1998). L'autonomie de l'élève et l'intégration des règles en éducation physique. Paris : PUF.

MINISTÈRE DE L'ÉDUCATION NATIONALE (2009). Programmes d'EPS pour les classes de lycée professionnel. B.O. spécial $n^{\circ} 2$ du 19 février.

Monnier, N., \& Amade-Escot, C. (2009). L'activité didactique empêchée : outil d'intelligibilité de la pratique enseignante en milieu difficile, Revue française de pédagogie 168, 59-73.

Rochex, J. Y. (1996). EPS interroge Jean - Yves Rochex : Rapport des jeunes au système éducatif aujourd'hui. Revue EPS, 262, 9-12, 96-98.

Schubauer-Leoni, M. L., \& Leutenegger, F. (2005). Une relecture des phénomènes transpositifs à la lumière de la didactique comparée, Revue suisse des sciences de l'éducation, 27 (3), 407-429.

Schubauer-Leoni, M. L. (2008). La construction de la référence dans l'action conjointe professeurélève. In N. Wallian, M. P. Poggi, \& M. Musard (Eds.), Co-construire des savoirs : les métiers de l'intervention par les APSA (pp. 67-86). Besançon : PUFC.

Schwartz, Y. (1997). Reconnaissance du travail. Pour une approche ergologique, Paris : PUF.

Sensevy, G., \& Mercier, A. (2007). Agir ensemble : Eléments de théorisation de l'action conjointe du professeur et des élèves. Rennes : PUR. 
Travert, M. (1997). Le "football de pied d'immeuble" : une pratique singulière au cœur d'une cité populaire, Ethnologie française, $\mathrm{n}^{\circ}$ XXVII, 188-196.

Zotian, E. (2010). « Les dimensions sociales de la pratique du football chez les garçons de milieux populaires ", in Actes du colloque Enfance et cultures : regards des sciences humaines et sociales, Sylvie Octobre et Régine Sirota (dir), [en ligne] http://www.enfanceetcultures.culture.gouv.fr/ actes/zotian.pdf, Paris.

\section{RÉSUMÉS}

L'objet de cet article est de présenter et d'analyser l'élaboration d'une forme de pratique scolaire du football en Education Physique et Sportive (EPS) inspirée de l'activité futsal et prenant en compte le rapport au sens construit par des élèves issus de milieu difficile. Notre étude concerne une classe de Terminale Baccalauréat Professionnel d'un lycée professionnel situé en "Zone Urbaine Sensible». La méthodologie reprend les trois phases caractéristiques de l'approche technologique à visée didactique (Bouthier \& Durey, 1994) : l'élaboration du projet, sa mise en œuvre et son évaluation. Elle prend appui sur des problématiques de terrain comme objet central d'étude (Bouthier, 2014). Notre recherche se centre sur l'identification et l'analyse de configurations de jeu, afin de qualifier les productions d'actions collectives des élèves et permettre une construction positive de la règle en EPS. Les résultats montrent que la coconstruction des savoirs est au cœur des transformations réelles et durables de ces élèves sur le plan de l'investissement et des apprentissages au niveau tactique et méthodologique.

The aim of our research is to present and to analyze the elaboration of a type of school practice for soccer in PE (Physical Education). It is inspired by the activity futsal and considers the building of meaning of pupils from a difficult background. Our study concerns a final year of a professional high school situated in "Sensitive Urban Zone". The methodology resumes three properties of technological approach with a didactic aim: the project elaboration, its implementation and evaluation (Bouthier \& Durey, 1994). It is based on professionnal issues as main aim (Bouthier, 2014). Our research speaks about identification and analysis of game configurations, to qualify pupils collective motor acquisition and enable a positive rule construction in PE. The results show the co-construction of knowledge turns out to be fundamental to lead real and long-lasting transformations of these pupils in their investment as well as in their learnings which can be both fundamental and methodological.

\section{INDEX}

Mots-clés : approche technologique, rapport au savoir, rapport à la règle, forme de pratique scolaire, football, configuration de jeu

Keywords : technological approach, relation of knowledge, relation of rule, type of school practice, soccer, game configuration

\section{AUTEUR}

\section{GUILLAUME DIETSCH}

Professeur Agrégé d'EPS - Lycée Professionnel Aristide Briand Le Blanc-Mesnil. Doctorant au LIRTES (EA7313), Université Paris-Est, France 